\title{
Saccharomyces cerevisiae fermentation products reduce bacterial endotoxin concentrations and inflammation during grain-based subacute ruminal acidosis in lactating dairy cows
}

\author{
J. Guo, ${ }^{1} \odot$ L. Xu, ${ }^{2 *}$ H. Khalouei, ${ }^{1}$ K. Fehr, ${ }^{1}$ V. Senaratne, ${ }^{1} \odot$ J. E. Ghia, ${ }^{4}$ I. Yoon, ${ }^{5} \odot$ E. Khafipour, ${ }^{1} \dagger \ddagger \odot$ \\ and J. C. Plaizier ${ }^{1} \ddagger \mathbb{D}$ \\ ${ }^{1}$ Department of Animal Science, University of Manitoba, Winnipeg, MB R3T 2E2, Canada \\ ${ }^{2}$ Department of Animal Science, Nanjing Agricultural University, Jiangsu, 210095, China ${ }^{3}$ Department of Immunology, University of Manitoba, \\ Winnipeg, MB R3E 0T5, Canada \\ ${ }^{4}$ Department of Internal Medicine, Section of Gastroenterology, University of Manitoba, Winnipeg, MB R3A 1R9, Canada \\ ${ }^{5}$ Diamond V, Cedar Rapids, IA 52404
}

\section{ABSTRACT}

Subacute ruminal acidosis (SARA) is a metabolic disorder in dairy cows that is associated with dysbiosis of rumen and hindgut microbiomes, translocation of immunogenic compounds from the gut lumen into blood circulation, and systemic inflammatory response. In this study we hypothesized that Saccharomyces cerevisiae fermentation products (SCFP) attenuate the increases in ruminal and peripheral bacterial endotoxin concentrations and the inflammation resulting from repeated induction of SARA. Lactating Holstein dairy cows (parity 2 and $3+, \mathrm{n}=32$ ) were fed diets with or without SCFP (all from Diamond V) and subjected to 2 episodes of SARA challenges. Cows received a basal total mixed ration (TMR) containing $34 \%$ neutral detergent fiber and $18.6 \%$ starch, dry matter $(\mathrm{DM})$ basis. Treatments were randomly assigned to control (basal TMR and $140 \mathrm{~g} / \mathrm{d}$ of ground corn with no SCFP) or 1 of 3 SCFP treatments: basal TMR and $14 \mathrm{~g} / \mathrm{d}$ Original XPC (SCFPa), $19 \mathrm{~g} / \mathrm{d}$ NutriTek $(\mathrm{SCFPb}-1 \times)$, or $38 \mathrm{~g} / \mathrm{d}$ NutriTek (SCFPb-2×) mixed with 126, 121, or $102 \mathrm{~g} / \mathrm{d}$ of ground corn, respectively. Treatments were implemented from 4 wk before until 12 wk after parturition. During wk 5 (SARA1) and wk 8 of lactation (SARA2), grain-based SARA challenges were conducted by gradually replacing $20 \%$ of DM of the basal TMR over $3 \mathrm{~d}$ with pellets containing $50 \%$ wheat and $50 \%$ barley. Ruminal fluid, fecal, and blood samples were collected weekly during Pre-SARA1 (wk 4, as baseline), Post-SARA1 (wk 7), and Post-SARA2 (wk

Received April 7, 2021.

Accepted November 13, 2021.

*Shuifa Agricultural Group Company Limited, Jinan, Shandong, 250100, China.

$\dagger$ Current address: Cargill Health Technologies, Wayzata, MN.

†Corresponding authors: Ehsan.Khafipour@gmail.com and Kees. Plaizier@ad.umanitoba.ca
10 for blood and wk 12 for rumen and fecal parameters) stages, and twice a week during the challenges SARA1 and SARA2. Rumen papillae samples were taken only during Pre-SARA1 and Post-SARA2. We measured the concentrations of free lipopolysaccharides (LPS) in the rumen fluid and feces; free LPS and lipoteichoic acid (LTA) endotoxins in peripheral plasma; interleukin (IL)-1 $\beta$ and IL-6 in peripheral serum; acute-phase proteins, serum amyloid A (SAA), and LPS-binding protein in peripheral plasma; haptoglobin $(\mathrm{Hp})$ in peripheral serum; and myeloperoxidase (MPO) in rumen papillae. Induction of SARA episodes increased free LPS concentrations in rumen fluid and tended to increase LTA in peripheral plasma. The SARA episodes increased concentration of circulating SAA and tended to increase that of IL-1 $\beta$ compared with Pre-SARA1. Induction of SARA did not affect the concentrations of circulating IL-6, Hp, and MPO. The SCFP supplementation reduced plasma concentrations of LTA and SAA and serum concentration of IL-1 $\beta$ compared with control. Additionally, SCFPb- $2 \times$ tended to reduce ruminal LPS in second-parity cows compared with control. Overall, SCFP supplementation appeared to stabilize the rumen environment and reduce proinflammatory status, hence attenuating adverse digestive and inflammatory responses associated with SARA episodes.

Key words: Saccharomyces cerevisiae fermentation products, SARA, endotoxins, inflammatory response, dairy cow

\section{INTRODUCTION}

A postbiotic is a "preparation of inanimate microorganisms and/or their components that confers a health benefit on the host" (Salminen et al., 2021). Examples of postbiotics used in dairy are Saccharomyces cerevisiae fermentation products (SCFP), which have been shown to benefit rumen fermentation (Zhu et al., 2017), 
milk production (Poppy et al., 2012), and efficiency of milk production of lactating dairy cows (Zhang et al., 2013). Supplementation with SCFP also have been shown to reduce inflammation during pathogenic (Vailati-Riboni et al., 2021), physiological (Li et al., 2016), and immunological challenges (Jiang et al., 2018). It is proposed that functional metabolites, organic acids, vitamins, and antioxidants present in postbiotics, such as SCFP, may either be used as nutrients by members of gut microbiota or act as signaling molecules affecting microbe-microbe or microbe-host interactions and the physiological and immunological responses of the host (Salminen et al., 2021).

Beneficial health effects of SCFP supplementation during SARA have been suggested (Li et al., 2016). This common metabolic disorder causes substantial economic loss to dairy farms and reduces the health and welfare of dairy cows (Krause and Oetzel, 2006; Plaizier et al., 2012); SARA has been associated with systemic inflammation (Krause and Oetzel, 2006) and dysbiosis of the rumen (Tun et al., 2020) and hindgut microbiota at the luminal, epimural, and mucosaassociated levels (Plaizier et al., 2021). Such dysbiosis changes the composition and reduces the functionality of rumen and hindgut microbiota by promoting proliferation of opportunistic bacteria (e.g., faster growers) and disintegration and lysis of those that cannot adapt to suboptimal rumen or hindgut environments (e.g., pH; Khafipour et al., 2016; Tun et al., 2020). As a result, endotoxins, such as LPS from gram-negative bacteria (Li et al., 2012; Khafipour et al., 2016), and perhaps lipoteichoic acid (LTA) from gram-positive bacteria (Weber et al., 2003), are released into the ruminal and intestinal digesta. These releases increase the concentration of luminal endotoxins and contribute to gut epithelial damage (Chin et al., 2006), particularly of the rumen papillae (Steele et al., 2009). The severity and frequency of SARA episodes dictate the magnitude of epithelial damage and the level of infiltration of immune cells, such as neutrophils and monocytes, in the rumen and intestinal tissues (Plaizier et al., 2012; Abbas et al., 2016; Khiaosa-ard and Zebeli, 2018). They also trigger release of reactive oxygen species and their catalyzers, such as myeloperoxidase (MPO) from these cells (Arroyo et al., 2017; Chami et al., 2018; Zhao et al., 2018). The combination of these events, accompanied by translocation of endotoxins and other immunogenic compounds out of the digestive tract and into peripheral blood circulation, may result in a systemic acute-phase protein (APP) response (Ginsburg, 2002; Eckel and Ametaj, 2016). This response may be driven by the release of proinflammatory cytokines, such as IL-1 $\beta$ and IL-6 (Trevisi et al., 2015). Hence, strategies that help stabilize conditions across the gastrointestinal tract, attenuate endotoxin release during SARA, prime the functionality of immune cells, and strengthen immune response may mitigate the adverse effects of SARA on health and production of lactating dairy cows.

A rich body of evidence suggests that SCFP supplementation is one such strategy. It has been demonstrated that SCFP stabilize the rumen microbial community (Tun et al., 2020), tend to reduce ruminal free LPS concentration, and enhance immune responses within and outside of the gut (Jensen et al., 2008; Perdomo Lozada, 2011; Li et al., 2012; Vailati-Riboni et al., 2021). As a result, we hypothesized that gut disruptions and inflammation during SARA can be attenuated by SCFP supplementation. The objectives of the study were, therefore, to determine the effects of 2 commercially available SCFP (XPC and NutriTek, Diamond V) on concentrations of LPS in the rumen and feces, concentrations of LPS and LTA of peripheral plasma, rumen papillae inflammatory status as determined by its MPO content, and systemic APP and cytokines responses of Holstein dairy cows subjected to 2 episodes of grain-induced SARA challenge during early lactation.

\section{MATERIALS AND METHODS}

This experiment was part of a larger randomized block design experiment conducted over 16 wk from 4 wk before to 12 wk after parturition. The protocol for the use of experimental animals was approved by the University of Manitoba Animal Care Committee (Winnipeg, Canada; protocol no. F14-038) and followed the guidelines of the Canadian Council for Animal Care (CCAC, 1993). Duration of ruminal pH below 5.6 from previous SCFP studies was used for power calculation. With a power of 0.80 and a type 1 error of 0.05 , a minimum of 6 replicates per treatment was required to identify a significant reduction in duration of ruminal $\mathrm{pH}$ below 5.6 as a result of SCFP supplementation. To account for potential loss of cows during the transition period, 8 replicates per treatment were included in the experiment. Here, we describe a subset of data on endotoxin and inflammatory responses during SARA challenges. Rumen production parameters and ruminal and total-tract digestibility data during SARA challenges are presented in Khalouei et al. (2021).

\section{Animals and Diets}

A total of 32 parity-2 $(\mathrm{n}=16)$ and multiparous (n $=16$ ) clinically healthy dairy cows were assigned into 
a randomized complete block design that contained 8 blocks. Cows were blocked based on parity, milk yield, and calving date. All cows were fixed with rumen cannulas at approximately $12 \mathrm{wk}$ before their expected calving date and were fully recovered from surgery before the start of the study, as evaluated by veterinarian staff. Cows within each block were randomly assigned to 1 of 4 treatments: with no supplementation (control), or supplemented with $14 \mathrm{~g} / \mathrm{d}$ Diamond V Original XPC (SCFPa, Diamond V), 19 g/d NutriTek (SCFPb-1×, Diamond V), or $38 \mathrm{~g} / \mathrm{d}$ NutriTek (SCFPb-2 $\times$, Diamond V) mixed with 140, 126, 121, and $102 \mathrm{~g} / \mathrm{d}$ of ground corn, respectively. The treatment diets were implemented from 4 wk before until 12 wk after parturition. The cows were fed individually, and SCFP was supplemented once daily as a top-dress.

\section{Experimental Design}

Cows were fed a prepartum diet with assigned treatment supplementation from the 4 wk prior to calving until parturition (Table 1). Following parturition, cows were fed the lactation diet containing $45 \%$ concentrate (DM basis) and their assigned treatment supplementation, except when SARA was induced. Grain-based SARA challenges were conducted during wk 5 (SARA1) and wk 8 (SARA2) of lactation by replacing $20 \%$ of the DM of the lactation TMR with pellets containing $50 \%$ ground wheat and $50 \%$ ground barley. Pellets were mixed with the rest of the TMR, and this replacement was completed gradually over $3 \mathrm{~d}$ before the beginning of SARA1 and SARA2. Detailed descriptions of feed sample collection and analyses are reported in our companion manuscript (Khalouei et al., 2021). The chemical and nutrient composition of diets are summarized in Table 1. During a 2-wk washout period between the 2 SARA challenges, cows were switched back to the lactation diet with treatment supplementation. Cows were fed the appropriate TMR ad libitum once daily at $0900 \mathrm{~h}$, with feed refusal allowance of $5 \%$ to $10 \%$. Cows had free access to fresh water during the experimental period. Rumen fluid, feces, and blood samples were collected once during the weeks of Pre-SARA1 (wk 4), Post-SARA1 (wk 7), and Post-SARA2 (blood samples on wk 10 and rumen and feces samples on wk 12) stages. During the SARA1 (wk 5) and SARA2 (wk 8) stages, samples were collected on the second and fifth day of the week (time points indicated as SARA1.1, SARA1.2, SARA2.1, and SARA2.2). All samples were collected $6 \mathrm{~h}$ after the $0900 \mathrm{~h}$ feed delivery. A schematic of the experimental design and sample collection is shown in Figure 1.

\section{Sample Collection and Analyses}

Sample Collection. Approximately $500 \mathrm{~mL}$ of whole rumen contents were collected into a $500-\mathrm{mL}$ bottle from 5 sites (cranial, caudal, dorsal, caudal ventral, and caudal dorsal) within the rumen and mixed. Solid and liquid digesta were separated using a Bodum coffee filter plunger (Bodum Inc.), as described by Petri et al. (2013). Briefly, $40 \mathrm{~mL}$ of rumen fluid was transferred into $50-\mathrm{mL}$ sterile centrifuge tubes and kept on ice until centrifugation at $12,000 \times g$ for $40 \mathrm{~min}$. Subsequently, the supernatant was filtered through a 0.22- $\mu \mathrm{m}$ LPS-free filter (Sigma, cat. no. Z359904) into a glass tube and baked at $100^{\circ} \mathrm{C}$ for $30 \mathrm{~min}(\mathrm{Li}$ et al., 2012). Samples were then stored at $-20^{\circ} \mathrm{C}$ for further LPS and LTA analyses.

Approximately $40 \mathrm{~g}$ of fecal grab samples were collected from the rectum after cleaning of the perineal area, placed into 50-mL sterile centrifuge tubes, and kept on ice. Briefly, $20 \mathrm{~g}$ of feces was mixed with equal amounts of physiological saline $(0.90 \% \mathrm{wt} / \mathrm{vol}$ of $\mathrm{NaCl})$, centrifuged, and processed as described previously for rumen samples. Filtered fecal samples were also kept at $-20^{\circ} \mathrm{C}$ for further LPS analysis.

To obtain papillae samples, rumens were partially emptied of their digesta during wk 4 and wk 10. Papillae samples were then clipped from 2 to 3 sites of the ventral sac, using a sterilized scissor. Samples were washed 10 times immediately after collection with ice-cold PBS ( $\mathrm{pH}$ 7.4) to remove the residual feed and gastrointestinal tract contents (Steele et al., 2009). Papillae samples (100 mg) were transferred into prelabeled $2-\mathrm{mL}$ vials, snap-frozen in liquid nitrogen, and stored at $-80^{\circ} \mathrm{C}$ for MPO detection.

Blood samples were taken at $6 \mathrm{~h}$ after feeding (1500 h) from the tail vein in plasma heparinized (143 US Pharmacopeia units) and blank serum evacuated tubes (Thermo Fisher Scientific). Briefly, $10 \mathrm{~mL}$ of sample in serum tubes were kept at room temperature for 30 min before centrifuging at $3,000 \times g$ at $4^{\circ} \mathrm{C}$ for $15 \mathrm{~min}$; from this, $1 \mathrm{~mL}$ of serum was stored at $-20^{\circ} \mathrm{C}$ for APP (haptoglobin, Hp) and cytokine (IL-6 and IL-1 $\beta$ ) analyses. The set of 10-mL blood samples in plasma tubes were immediately centrifuged at $3,000 \times g$ at $4^{\circ} \mathrm{C}$ for $15 \mathrm{~min}$. Subsequently, $1 \mathrm{~mL}$ of plasma was transferred into pyrogen-free glass tubes and stored at $-20^{\circ} \mathrm{C}$ for LPS and LTA analyses. Another $1 \mathrm{~mL}$ of plasma was stored at $-20^{\circ} \mathrm{C}$ for APP serum amyloid A (SAA) and LPS-binding protein (LBP) measurements.

LPS and LTA Analyses. Concentrations of LPS in rumen fluid and feces were determined by a chromogenic kinetic Limulus amebocyte lysate assay (Kinetic-QCL, 
cat. no. 50-650u) using a 50\% $\beta$-glucan blocker (cat. no. N190; both from Lonza Group Ltd.) as previously described (Khafipour et al., 2009). Blood plasma, rumen fluid, and feces were diluted with Limulus amebocyte lysate reagent water (cat. no. W50-500), $10 \mathrm{mM} \mathrm{MgCl}$ solution (cat. no. S50-641), and Pyrosperse (cat. no. N188; all from Lonza Group Ltd.). Dilution rates were 1:80,943 for rumen fluid, 1:50,719 for feces, and 1:28 for blood plasma. To verify the lack of LPS inhibition, samples were spiked with $3 \mu \mathrm{L}$ of 5 endotoxin units $(\mathbf{E U}) / \mathrm{mL}$ commercial Escherichia coli LPS standard (Lonza Group Ltd.). A recovery range between 50 and $150 \%$ of the spiked concentration was considered as noninhibitory dilution, as suggested by the manufacturer. As our recovery rates were $83.3 \% \pm 0.04 \%$ (mean $\pm \mathrm{SD}$ ) for rumen liquid and $92.4 \% \pm 0.03 \%$ for feces, the kits performed satisfactorily.

The concentrations of LTA in rumen liquid and blood plasma were determined using an ELISA assay with a commercial kit (OKEH02591, Aviva Systems Biology) according to manufacturer's instructions. Rumen liq- uid samples were diluted from 0 to $10^{-6}$; blood plasma samples were analyzed without dilution.

Optical densities were determined at $405 \mathrm{~nm}$ for the LPS assay and $450 \mathrm{~nm}$ for the LTA assay, as per kits' instructions, using a microplate spectrophotometer (Synergy H4 System, BioTek Instruments Inc.).

Measures of Inflammatory Response. For measurement of SAA and LBP, blood plasma samples were diluted 1:500 with distilled water. Diluted samples were then processed using a commercial ELISA kit for SAA (cat. no. TP802, Tridelta Development Ltd.) and LBP (cat. no. HK503, Hycult Biotech), as described by Khafipour et al. (2009). The Hp concentration in blood serum was determined with a commercial ELISA kit (cat. no. TP801, Tridelta Development Ltd.) following the manufacturer's instructions. All optical densities were determined using the recommended wavelengths by the kit manufacturers, using a microplate spectrophotometer (Synergy H4 System, BioTek Instruments Inc.). The recommended wavelengths were $450 \mathrm{~nm}$ for SAA and LBP and $630 \mathrm{~nm}$ for Hp. Optical densities

Table 1. Ingredient and chemical compositions of experimental diets

\begin{tabular}{|c|c|c|c|}
\hline Item & $\begin{array}{l}\text { Close-up dry } \\
\text { cow TMR }\end{array}$ & $\begin{array}{l}\text { Lactating } \\
\text { TMR }\end{array}$ & $\begin{array}{l}\text { SARA } \\
\text { TMR }\end{array}$ \\
\hline \multicolumn{4}{|l|}{ Ingredient composition (\% of DM) } \\
\hline Round bale mixed alfalfa/grass silage & 20 & 35 & 28 \\
\hline Alfalfa first-cut haylage & 8 & - & - \\
\hline Corn silage & 37 & - & - \\
\hline Barley silage & - & 20 & 16 \\
\hline Straw & 13 & — & \\
\hline Ground corn & - & 20 & 16 \\
\hline Dairy Aide ${ }^{1}$ & & 25 & 20 \\
\hline Close-up dry cow supplement ${ }^{2}$ & 22 & - & - \\
\hline Wheat-barley pellets & - & - & 20 \\
\hline \multicolumn{4}{|l|}{ Chemical composition (\% of DM) } \\
\hline $\mathrm{DM}(\%)$ & 48 & 51 & 60 \\
\hline $\mathrm{CP}$ & 15.5 & 17.9 & 17.2 \\
\hline Fat & 2.8 & 4.3 & 3.6 \\
\hline Starch & 17.6 & 18.6 & 27.9 \\
\hline NDF & 38.7 & 34.9 & 28.2 \\
\hline $\mathrm{ADF}$ & 26.7 & 26.0 & 19.9 \\
\hline $\mathrm{Ca}$ & 1.26 & 1.32 & 1.06 \\
\hline $\mathrm{P}$ & 0.37 & 0.45 & 0.47 \\
\hline $\mathrm{Mg}$ & 0.41 & 0.37 & 0.34 \\
\hline $\mathrm{Na}$ & 0.06 & 0.33 & 0.27 \\
\hline $\mathrm{K}$ & 2.37 & 2.57 & 2.02 \\
\hline
\end{tabular}

${ }^{1}$ Dairy Aide (Trouw Nutrition) contained flaked corn $(65.2 \%)$, corn distillers grain $(7.1 \%)$, APF Fat Plus (3.6\%; Auburn Laboratories Inc.), feather meal (3.6\%), porcine meat meal (2.9\%), soybean meal $(2.9 \%)$, canola meal (2.9\%), AV Fat Rothsay Feeders Choice (2.2\%; Rothsay), sodium sesquicarbonate (1.9\%; SQ 810, Arm \& Hammer Animal Nutrition), dicalcium phosphate (1.5\%), potassium chloride (1.5\%; DYNA K Red, Prince Agri Products Inc.), ground limestone (1.5), salt-potash (1.5\%), Dairy LMK Ultra Micro (0.6\%; Trouw Nutrition), magnesium oxide $(0.3 \%)$, methionine analog ( $0.3 \%$; Novus).

${ }^{2}$ Dry cow supplement contained Landmark close-up dry cow pellets $(50.0 \%)$, flaked corn (20.0\%), beet pulp pellets $(15.0 \%)$, rolled barley $(12.5 \%)$, liquid molasses $(2 \%)$, soy oil $(0.5 \%)$, liquid caramel $(0.01 \%)$. Landmark close-up day cow pellets contained barley $(4.0 \%)$, limestone $(7.0 \%)$, corn distillers grain $(34.9 \%)$, dicalcium phosphate $(1.8 \%)$, canola meal $(23.0 \%)$, soybean meal $(8.5 \%)$, wheat $(15.0 \%)$, niacin $(0.3 \%)$, Biopowder SXC (0.05\%; Lallamand), magnesium oxide (1.6\%), transition VB 25K (2.9\%; Jefo Nutrition), dry cow MicroPX premix (1\%; Friesen Nutrition). 


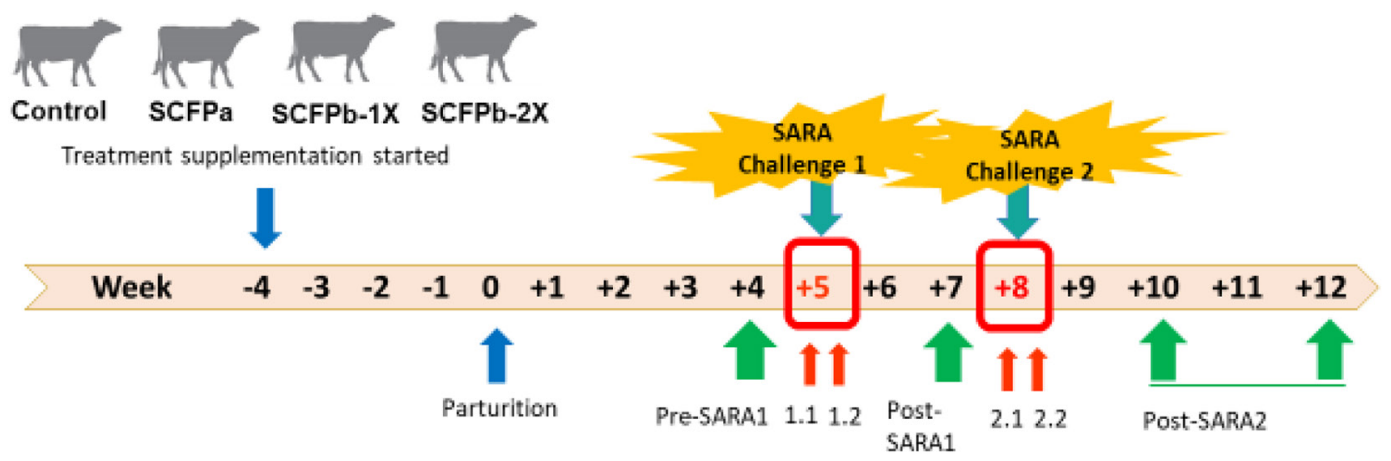

Figure 1. Experimental design. A total of 32 cows were randomly divided into 4 treatment groups with 8 cows per group based on parity, level of production, and calving date. Treatments were as follows: control $=140 \mathrm{~g} / \mathrm{d}$ ground corn; SCFPa $=14 \mathrm{~g} / \mathrm{d}$ Diamond V Original XPC mixed with $126 \mathrm{~g} / \mathrm{d}$ ground corn; SCFPb- $1 \times=19 \mathrm{~g} / \mathrm{d}$ NutriTek (Diamond V) mixed with $121 \mathrm{~g} / \mathrm{d}$ ground corn; SCFPb-2× = 38 g/d NutriTek mixed with $102 \mathrm{~g} / \mathrm{d}$ ground corn. SARA was induced during wk 5 (SARA1) and 8 (SARA2) post-parturition. Week 4 was considered as PreSARA1, wk 7 as Post-SARA1, and wk 10 or 12 (depending on the parameter) as Post-SARA2. Samples were collected weekly, with the exception of challenge periods, during which sampling was performed on d 2 and 5 of each SARA challenge week (SARA1.1, SARA1.2, SARA2.1, and SARA2.2).

from negative controls and 6 dilutions of positive controls were used for development of a standard curve and calculation of SAA, LBP, and HP in samples.

Serum IL-6 and IL-1 $\beta$ concentrations were determined with a Bovine IL-6 ELISA Reagent Kit (ESS0029) and a Bovine IL-1 $\beta$ ELISA Reagent Kit (ESS0027; both from Thermo Fisher Scientific), respectively. Additional ELISA reagent kit buffers used included DPBS (28374), carbonate-bicarbonate buffer (28382), blocking buffer (N502), wash buffer (N503), and BSA (A4737-1G; all from Thermo Fisher Scientific). Optical densities for IL-6 and IL-1 $\beta$ assays were read under recommended 450-nm wavelengths using a microplate spectrophotometer (Synergy H4 System, BioTek Instruments Inc.). The recovery range was 82 to $93 \%$ for IL- 6 and 96 to $116 \%$ for IL-1 $\beta$, respectively.

Myeloperoxidase Concentration in Rumen Papillae. Frozen papillae samples that had been stored at $-80^{\circ} \mathrm{C}$ were ground in liquid nitrogen with a mortar. Briefly, $50 \mathrm{mg}$ of ground samples were transferred into 2 -mL centrifuge tubes with $1 \mathrm{~mL}$ of fresh lysis buffer (IS007-BUF3, Cloud-Clone Corp.) and sonicated with an ultrasonic processor (VC 130, Sonics \& Materials Inc.). Subsequently, MPO concentration was determined using a Bovine MPO ELISA kit (SEA601Bo, Cloud-Clone Corp.) according to the manufacturer's instructions. Optical densities were read immediately at $450 \mathrm{~nm}$ using a microplate spectrophotometer (Synergy H4 System, BioTek Instruments Inc.). The recovery range was 80 to $102 \%$. Total protein concentration was determined using a Pierce BCA protein assay kit (SF249046, Thermo Fisher Scientific). The MPO concentration was expressed as nanograms of total protein.

\section{Statistical Analysis}

Normality of distributions of residuals for LPS, LTA, APP, and cytokine data was tested using the UNIVARIATE procedure of SAS (version 9.4, SAS Institute Inc.) by Shapiro-Wilk's statistic. If the residuals were not normal, the data were either $\log _{10}$ transformed or raised to the power of lambda to achieve normality (Tables 2, 3,4 , and 5). Lambda was calculated by Box-Cox transformation using the TRANSREG procedure of SAS. Original or transformed data were then analyzed by the MIXED procedure of SAS using the following model:

$$
\begin{aligned}
\mathrm{Y}_{\mathrm{ijk}}= & \mu+\mathrm{T}_{\mathrm{i}}+\mathrm{S}_{\mathrm{j}}+\mathrm{P}_{\mathrm{k}}+(\mathrm{T} \times \mathrm{S})_{\mathrm{ij}}+(\mathrm{T} \times \mathrm{P})_{\mathrm{ik}} \\
& +(\mathrm{P} \times \mathrm{S})_{\mathrm{kj}}+(\mathrm{T} \times \mathrm{P} \times \mathrm{S})_{\mathrm{ijk}}+\mathrm{e}_{\mathrm{ijk}} .
\end{aligned}
$$

The variable $Y_{i j k}$ was dependent on $\mu$ as the average experimental value and fixed effects of treatment $T_{i}$ $(\mathrm{i}=$ control, SCFPa, SCFPb- $1 \times$, and SCFPb- $2 \times$ ), stage $S_{j}(j=$ Pre-SARA1, SARA1, Post-SARA1, SARA2, and Post-SARA2), parity $\mathrm{P}_{\mathrm{k}}(\mathrm{k}=$ parity 2 or $3+)$, and interactions $(\mathrm{T} \times \mathrm{S})_{\mathrm{ij}},(\mathrm{T} \times \mathrm{P})_{\mathrm{ik}},(\mathrm{P} \times$ $\mathrm{S})_{\mathrm{kj}}$, and $(\mathrm{T} \times \mathrm{S} \times \mathrm{P})_{\mathrm{ijk}}$. Data from the second and fifth days of each SARA week (time points SARA1.1, SARA1.2, SARA2.1, and SARA2.2) were combined as SARA1 and SARA2, as no difference was observed between SARA1.1 and SARA1.2 or between SARA2.1 and SARA2.2. Level of LTA was measured only during SARA1, and SARA1.1 and SARA1.2 data were presented separately. Block was considered as random, and stage was considered as a repeated measure. The PDIFF option was applied for pairwise comparisons 
between treatments, parities, stages, and their interactions. Comparison of least squares means was adjusted with the Tukey test. Contrast comparisons were made between control and SCFP, SCFPa and SCFPb, and $\mathrm{SCFPb}-1 \times$ and $\mathrm{SCFPb}-2 \times$. All analyses were initially performed with the MIXED model described herein. If the effect of parity was not significant or no trend was observed, parity and its interactions were removed from the model (Tables 2, 3, 4, and 5).

Results are presented as least squares means with their pooled standard errors. For results interpretation, the data that were transformed to achieve normality are presented as means from the original data within tables (Tables 2, 3, 4, and 5). Significant effects were considered at $P<0.05$, and tendencies were discussed at $0.05 \leq P<0.1$.

\section{RESULTS}

The health of animals was monitored daily. During the larger study 2 cows died, one from the SCFPb- $2 \times$ group, which died during the prepartum period due to complications of cannulation surgery, and the other from the SCFPb-1× group, which died following the first SARA challenge. Samples from these cows were removed from the data set. Additionally, one cow in the SCFPa group during the transition period and one cow in SCFPb-1× after the second SARA challenge were diagnosed with mastitis. Also, one cow in the SCFPb- $2 \times$ group was diagnosed with milk fever during the transition period; however, she recovered before SARA1 was conducted. Data from these cows during the indicated time points were removed from the statistical analysis.

\section{Effects of SARA on Bacterial Endotoxin (LPS and LTA) Concentrations in the Rumen, Feces, and Plasma}

Pooled across SCFP treatments, the SARA challenges compared with pre- and post-challenge stages increased the concentration of free LPS in the rumen fluid (66,140 vs. $5,215 \mathrm{EU} / \mathrm{mL}, P<0.001)$ and in the feces $(81,628$ vs. $8,221 \mathrm{EU} / \mathrm{mL}, P<0.01$; Table 2$)$. The free LPS concentrations in rumen fluid and feces did not differ between the 2 SARA challenge stages, or among the Pre-SARA1, Post-SARA1, and PostSARA2 stages (Table 2). The LPS concentrations in the plasma samples were below the detection limit of the assay $(<0.005 \mathrm{EU} / \mathrm{mL})$ during all stages. The SARA challenges tended to increase $(P=0.07)$ plasma LTA concentrations compared with Pre-SARA1 (0.70 vs. $0.65 \mathrm{ng} / \mathrm{mL}$; Table 3 ).

\section{Effects of SCFP on Bacterial Endotoxin (LPS and LTA) Concentrations in the Rumen, Feces, and Plasma}

The SCFP treatments did not affect ruminal and fecal free LPS concentrations before, after, or during SARA challenges. However, parity and interaction of parity and SCFP treatments tended to affect $(P$ $=0.06$ ) rumen free LPS (Table 2 ). In parity-2 cows, pooled across Pre-SARA, Post-SARA, and SARA stages, $\mathrm{SCFPb}-2 \times$ tended to reduce $(P=0.077)$ rumen free LPS concentrations compared with control $(6,670$ vs. 13,182 EU/mL). The LPS concentrations in plasma samples remained undetected for all treatments.

The concentration of LTA was affected by SCFP treatment $(P=0.006)$ and parity $(P=0.03)$, but not by any of the interactions (Table 3 ). Pooled across Pre-SARA1 stage and SARA challenge stages, the SCFPb- $1 \times$ treatment reduced $(P=0.006)$ and $\mathrm{SCFPb}-2 \times$ tended to reduce $(P=0.07)$ plasma concentrations of LTA compared with control $(0.63$ and 0.65 vs. $0.73 \mathrm{ng} / \mathrm{mL}$, respectively). Contrast comparisons showed a reduced plasma LTA in SCFP compared with control (0.66 vs. $0.73 \mathrm{ng} / \mathrm{mL} ; P=0.008)$ and in SCFPb compared with SCFPa (0.64 vs. $0.71 \mathrm{ng} / \mathrm{mL} ; P=0.02)$. Average across SCFP treatments and SARA stages, parity- 2 cows had greater $(P=0.03)$ plasma LTA compared with parity-3+ cows (0.72 vs. $0.64 \mathrm{ng} / \mathrm{mL}$ ).

\section{Effects of SARA on Inflammatory Responses}

Regardless of SCFP treatments, the SARA challenges increased $(P<0.01)$ the blood plasma concentrations of SAA compared with Pre-SARA1 (63.38 vs. $19.76 \mu \mathrm{g} /$ $\mathrm{mL})$ but did not affect that of Hp (129.03 vs. $81.61 \mu \mathrm{g} /$ $\mathrm{mL}$ ) or LBP (11.86 vs. $11.16 \mu \mathrm{g} / \mathrm{mL}$; Table 4 ). Compared with SARA1, plasma LBP concentration tended to be lower $(P=0.07)$ during Post-SARA1 (10.01 vs. $12.77 \mu \mathrm{g} / \mathrm{mL})$ and was reduced $(P<0.01)$ during PostSARA2 (8.99 vs. $12.77 \mu \mathrm{g} / \mathrm{mL})$. The SARA challenges did not affect the blood plasma concentration of cytokine IL-6, regardless of SCFP treatments (Table 4). Pooled across SCFP treatments, plasma IL-1 $\beta$ concentration tended to increase $(P=0.07)$ from Pre-SARA1 to Post-SARA2 (4.51 vs. $8.71 \mathrm{pg} / \mathrm{mL}$ ). We found no difference between the 2 SARA challenges in all parameters. The MPO concentration in rumen papillae did not differ between the Pre-SARA1 and Post-SARA2 stages (Table 5).

\section{Effects of SCFP on Inflammatory Responses}

Pooled across Pre-SARA, Post-SARA, and SARA stages, SCFP treatments affected the blood plasma 


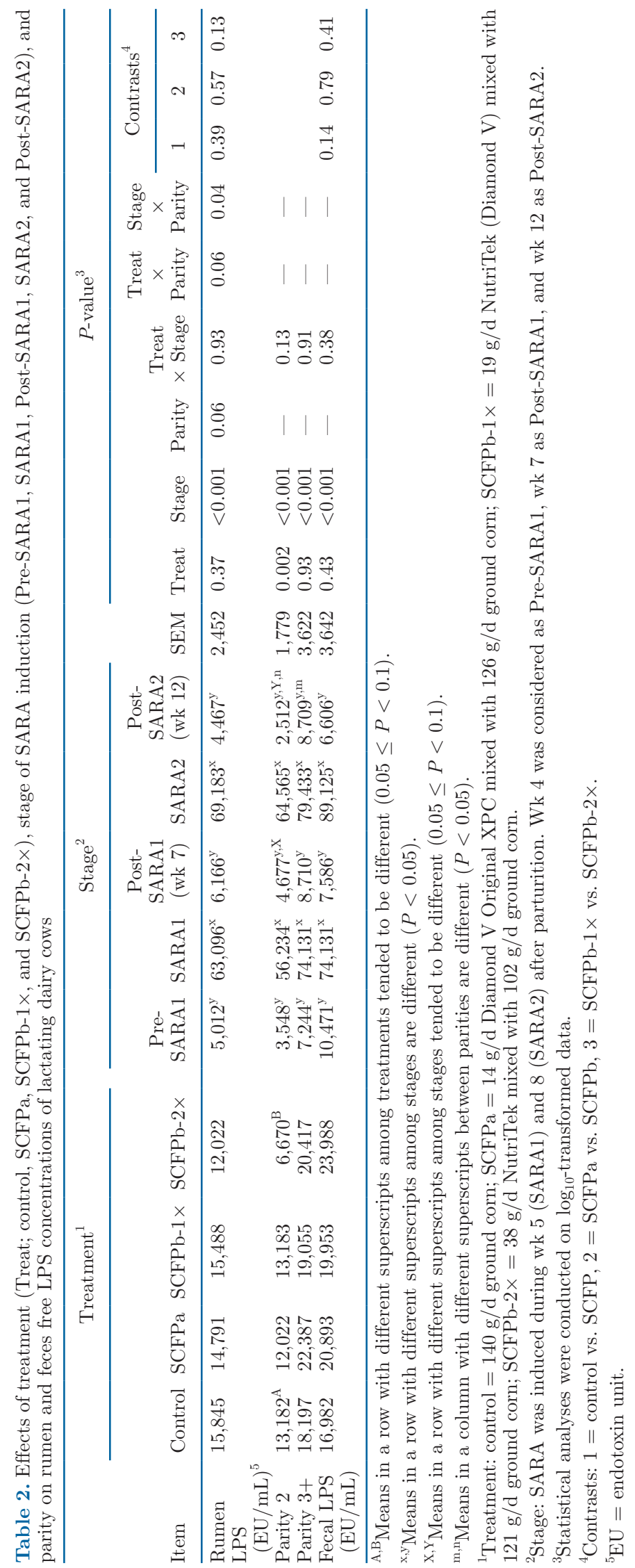


concentrations of SAA and LBP, and blood serum concentrations of Hp, IL-1 $\beta$, and IL-6 (Table 4). The interaction between stage and SCFP treatments was not significant for any of these variables. Feeding SCFP resulted in reduced $(P=0.02)$ plasma SAA concentrations compared with the control group (27.87 vs. $39.81 \mu \mathrm{g} / \mathrm{mL}$ ). This reduction was more pronounced in $\mathrm{SCFPb}-2 \times$ cows compared with control cows $(23.14$ vs. $39.81 \mu \mathrm{g} / \mathrm{mL} ; P=0.05)$. Supplementation wih $\mathrm{SCFPb}$ reduced serum Hp concentrations compared with SCFPa (90.51 vs. $141.74 \mu \mathrm{g} / \mathrm{mL} ; P=0.05)$. Between $\mathrm{SCFPb}$ treatments, $\mathrm{SCFPb}-2 \times$ resulted in more reduction $(P=0.03)$ in $\mathrm{Hp}$ compared with $\mathrm{SCFPb}-1 \times$ $(63.30$ vs. $117.73 \mu \mathrm{g} / \mathrm{mL})$. Regardless of SCFP type, SCFP feeding tended to reduce $(P=0.09)$ plasma LBP compared with control (10.40 vs. $11.68 \mu \mathrm{g} / \mathrm{mL}$ ). In particular, SCFPa supplementation reduced plasma LBP concentrations compared with control (9.22 vs. $11.68 \mu \mathrm{g} / \mathrm{mL} ; P=0.05)$ and $\mathrm{SCFPb}(9.22$ vs. 11.04 $\mu \mathrm{g} / \mathrm{mL} ; P=0.03)$. The effects of SCFPa compared with $\mathrm{SCFPb}$ were more pronounced in parity-3+ cows compared with parity-2 cows.

Pooled across Pre-SARA, Post-SARA, and SARA stages, SCFP supplementation reduced $(P<0.001)$ blood serum IL-1 $\beta$ concentrations compared with control (5.25 vs. $11.94 \mathrm{pg} / \mathrm{mL})$. The reduction in serum IL$1 \beta$ concentrations was greater in SCFPa than $\mathrm{SCFPb}$ $(4.13$ vs. $6.58 \mathrm{pg} / \mathrm{mL} ; P=0.03)$ and in SCFPb- $2 \times$ compared with SCFPb- $1 \times(3.71$ vs. $9.45 \mathrm{pg} / \mathrm{mL} ; P<$ 0.001). Similar to IL-1 $\beta$, SCFPa-fed cows had lower $(P$ $=0.0$ ) serum IL-6 concentration compared with SCFPb cows (256.39 vs. $431.02 \mathrm{ng} / \mathrm{mL}$ ). Average across SCFP treatments and SARA stages, parity-2 cows had greater $(P<0.01)$ serum IL-1 $(17.48$ vs. $3.65 \mathrm{pg} / \mathrm{mL})$ and IL-6 concentrations (635.97 vs. $216.19 \mathrm{pg} / \mathrm{mL}$ ) compared with parity-3+ cows.

The MPO concentration in rumen papillae did not differ between the Pre-SARA1 and Post-SARA2 stages or among SCFP treatments (Table 5).

\section{DISCUSSION}

Postbiotics from Saccharomyces cerevisiae fermentation contain a range of bioactive compounds that stabilize rumen microbiota and reduce inflammatory response, resulting in improved gut health and immune balance (Jensen et al., 2008; Li et al., 2016; Tun et al., 2020). In this study, we tested whether 2 commercially available SCFP (SCFPa, and SCFPb at $1 \times$ and $2 \times$ doses) can ameliorate the disrupted gut and immune health of dairy cows subjected to SARA challenges. We demonstrated that supplementation of SCFPb at the $2 \times$ dose attenuated free LPS concentration in the rumen fluid in parity- 2 cows. We further showed that 
Guo et al.: SCFP ATTENUATES NEGATIVE EFFECTS OF SARA

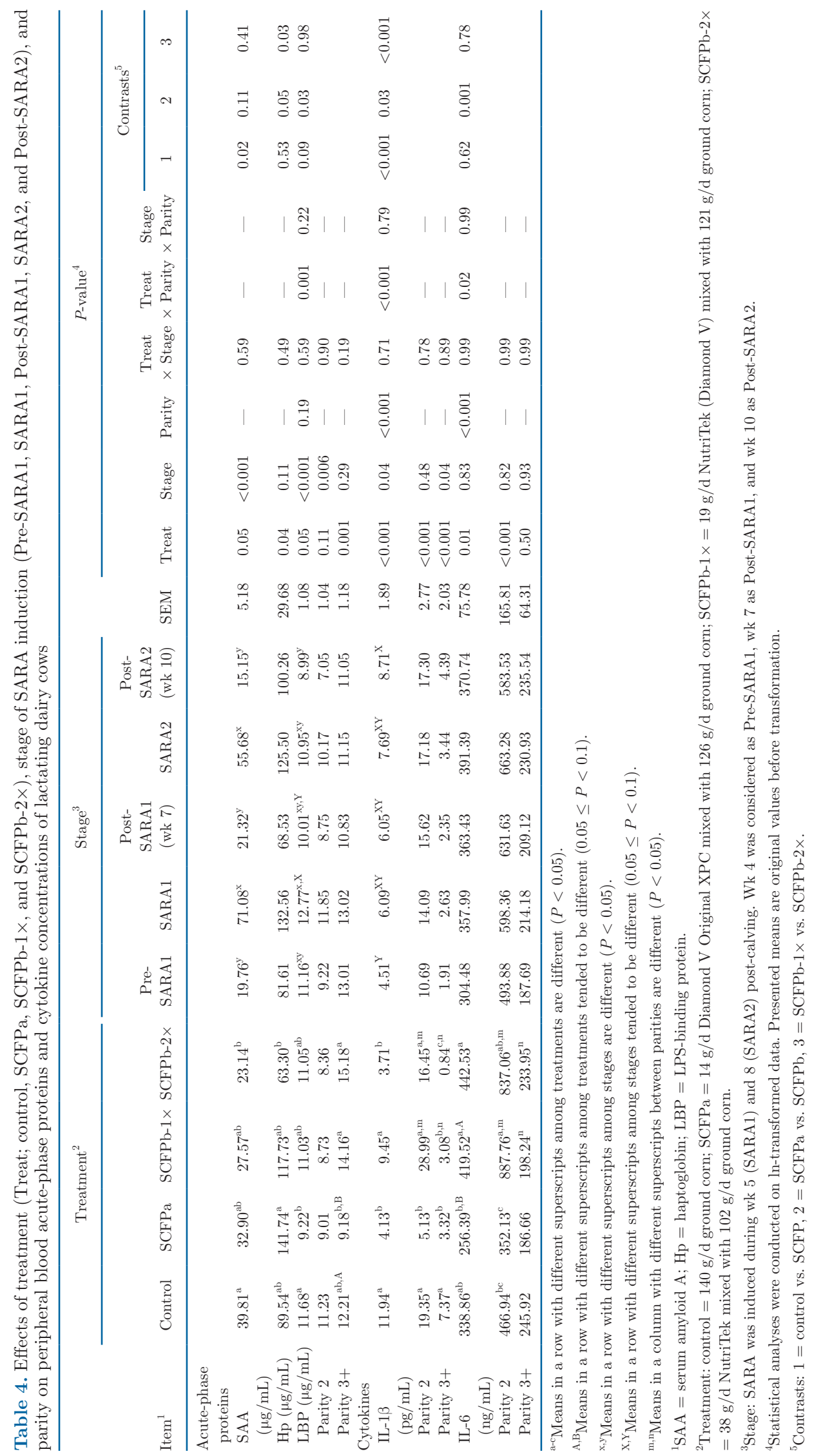


supplementation of $\mathrm{SCFPb}$ at both $1 \times$ and $2 \times$ doses attenuated plasma concentration of LTA. This perhaps was an indicator of either a reduced translocation of LTA endotoxins from the gut lumen and, hence, improved gut epithelial integrity, or greater capacity of the immune system for clearing LTA from the systemic circulation. Also, SCFPb supplementation at the $2 \times$ dose reduced plasma concentration of SAA and IL-1 $\beta$ compared with control-fed cows, indicating a reduced proinflammatory status, perhaps due to a priming effect of SCFP. Additionally, SCFPb supplementation showed more effect on inflammatory parameters compared with SCFPa, which could be due to its higher content of antioxidants and polyphenol compounds (Khalouei et al., 2021).

\section{Assessment of SARA Induction Protocol}

In our study, SARA was defined as daily episodes of rumen $\mathrm{pH}$ between 5.2 and 5.6 for at least $180 \mathrm{~min}$ per day (Gozho et al., 2007). These thresholds are important, in that at least $3 \mathrm{~h}$ per day of $\mathrm{pH}$ below 5.6 is required to provoke an inflammatory response (Gozho et al., 2007). The companion paper from Khalouei et al. (2021) reported that, across treatments, the SARA challenge increased the duration of rumen $\mathrm{pH}$ below 5.6 from 8 to $186 \mathrm{~min} / \mathrm{d}$. Hence, on average, the duration of rumen $\mathrm{pH}$ below 5.6 during the SARA challenge was only $6 \mathrm{~min} / \mathrm{d}$ longer than the threshold. As this duration did not greatly exceeded the SARA threshold, it was expected that a less severe inflammatory response or gut disturbance would be observed, compared with previously reported grain-induced SARA challenges with durations longer than the threshold (e.g., $100 \mathrm{~min}$ longer in Khafipour et al., 2009, or 200 min longer in Zhao et al., 2018).

\section{Effect of SARA on LPS Concentrations in Rumen Fluid and Feces}

The observed increases in ruminal free LPS concentrations were lower than those reported in the literature during grain-based SARA challenges (e.g., from 24,547 to 128,820 in Gozho et al., 2007; from 30,768 to 130,589 in Zhao et al., 2018; and from 14,581 to $168,285 \mathrm{EU} /$ $\mathrm{mL}$ in Humer et al., 2018). This confirms that moderate $\mathrm{pH}$ depression in our study resulted in moderate increases in ruminal free LPS. In contrast, the observed increases in fecal free LPS were similar to previously reported increases in the literature (e.g., from 12,832 to 93,154 and from 29,679 to $77,371 \mathrm{EU} / \mathrm{g}$ of wet feces in $\mathrm{Li}$ et al., 2012, and $\mathrm{Li}$ et al., 2016). In contrast, Qumar et al. (2017) reported greater concentrations of free LPS for both baseline and SARA, from 133,165 
to $1,049,198$ during an abrupt SARA induction similar to our study, and from 175,245 to $1,256,181 \mathrm{EU} / \mathrm{g}$ wet feces when SARA was continuously induced for 2 wk. The concentration of free LPS in the feces is a function of carbohydrates bypassed from the rumen to the large intestine ( $\mathrm{Li}$ et al., 2012). A survey (Li et al., 2010) of 300 lactating dairy cows on 10 farms reported that fecal free LPS ranged from 3,514 to 252,345 with a median of 34,982 and 75 th percentile of $63,179 \mathrm{EU} / \mathrm{g}$ of wet feces. In that survey, the variations in fecal free LPS were mostly explained by the amount of dietary NDF among farms, DIM, and plasma Hp concentration among cows. Considering the evidence, the concentrations reported in the study of Qumar et al. (2017) were perhaps on the higher end, and our SARA challenges successfully increased fecal LPS to the ranges that are usually expected during SARA. Additionally, when free ruminal and fecal LPS between the 2 SARA challenges were compared, SARA1 and SARA2 did not differ in these LPS contents, confirming the consistency of our induction model (Table 2; Supplemental Table S1, https:/ /figshare.com/articles/online_resource/Supplemental _data_for_DOI_10_3168_jds_2021-20572/17162603, Guo et al., 2021). Also, a lack of difference in ruminal or fecal free LPS contents among Pre-SARA1, PostSARA1, and Post-SARA2 was indicative that cows returned to the baseline (Pre-SARA1) within 2 to $4 \mathrm{wk}$ after SARA challenge.

\section{Effect of SCFP on LPS Concentrations in Rumen Fluid and Feces}

Within parity- 2 cows, SCFPb- $2 \times$ supplementation tended to reduce ruminal free LPS concentrations compared with the control group $(P=0.077)$ for both the Pre-SARA1 $(1,862 \mathrm{EU} / \mathrm{mL})$ and pooled across SARA weeks $(46,707 \mathrm{EU} / \mathrm{mL}$; Table 2; Supplemental Table S2, https://figshare.com/articles/online_resource/ Supplemental_data_for_DOI_10_3168_jds_2021 -20572/17162603, Guo et al., 2021). The rate of LPS release from gram-negative bacteria is a function of availability of nutrients and $\mathrm{pH}$ (Hurley, 1995). The availability of highly degradable nutrients in the rumen promotes proliferation of bacteria and, hence, release of LPS during the logarithmic and stationary phases of bacterial growth, while bacteria are expanding their cell walls. Suboptimal rumen $\mathrm{pH}$, however, results in disintegration and lysis of bacterial cell walls and release of LPS from organisms that cannot tolerate the lower pH (Plaizier et al., 2012; Khafipour et al., 2016; Monteiro and Faciola, 2020). A reduction of ruminal free LPS concentration hence may be an indicator that $\mathrm{SCFPb}-2 \times$ stabilized the rumen microbial community by controlled proliferation of gram-negative bacterial taxa, such as members of Proteobacteria and Bacteroidetes phyla, which are major contributors to the rumen free LPS pool (Mao et al., 2013; Khafipour et al., 2016; Guo et al., 2018). This hypothesis is supported by ruminal microbiome data showing that SCFPb- $2 \times$ promoted microbiome stability by ameliorating the negative effects of SARA on community evenness and abundances of foundation members (Guo et al., 2018). The $\mathrm{SCFPb}-2 \times$ treatment also stabilized the rumen $\mathrm{pH}$ environment $(79.3 \mathrm{~min} / \mathrm{d}$ of $\mathrm{pH}<5.6)$ by switching the site of starch digestion from the rumen to the intestines (Khalouei et al., 2021), which perhaps contributed to a lower rumen LPS concentration compared with the control group.

\section{Effects of SARA and SCFP on LPS and LBP Concentrations in Peripheral Blood Plasma}

Regardless of the high-sensitivity assay used, peripheral plasma LPS concentration remained below our detection limit of $0.005 \mathrm{EU} / \mathrm{mL}$ for all samples. This suggests that the rate of LPS translocation through the rumen or hindgut wall was perhaps below the rate of LPS clearance by the liver (Haubro Andersen and Jarlov, 1990). Therefore, LPS did not accumulate in the peripheral plasma above the detection limit of the assay. Plasma LBP concentration also did not differ among Pre-SARA1, SARA1, and SARA 2. The only tendencies or significant differences observed in LBP concentration were among SARA1, Post-SARA1, and Post-SARA2 (Table 4; Supplemental Tables S3 and S4, https://figshare.com/articles/online_resource/ Supplemental_data_for_DOI_10_3168_jds_2021 $-20572 / 17162603$, Guo et al., 2021). As LBP is an APP that only binds to LPS and facilitates its transfer to membrane-associated receptors, its greater concentrations are indicative of LPS trafficking from the gut lumen into circulation (Eckel and Ametaj, 2016). In this study, however, the plasma concentration of LBP during SARA was lower than that of previous reports (e.g., 11.86 vs. $53.2 \mu \mathrm{g} / \mathrm{mL}$ in Khafipour et al., 2009, or $40.9 \mu \mathrm{g} / \mathrm{mL}$ in Zhao et al., 2018), where authors induced a more severe rumen $\mathrm{pH}$ depression below 5.6 (279 and $369 \mathrm{~min} / \mathrm{d}$, respectively) compared with current study $(186 \mathrm{~min} / \mathrm{d})$ and were able to detect LPS in plasma (0.52 and $0.21 \mathrm{EU} / \mathrm{mL}$, respectively).

\section{Effects of SARA and SCFP on LTA Concentrations in Peripheral Blood Plasma}

The SARA challenge resulted in a tendency for greater LTA on both the second (SARA1.1) and the 
fifth day (SARA1.2) of SARA compared with that of Pre-SARA1, indicating greater translocation of LTA during the challenge week (Table 3; Supplemental Table S5, https://figshare.com/articles/online_resource/ Supplemental_data_for_DOI_10_3168_jds_2021 -20572/17162603, Guo et al., 2021). That being said, the presence of a detectable amount of LTA in peripheral circulation during Pre-SARA1 (0.65 ng/mL) was interesting and was an indication of weaker immune response and more tolerance to LTA compared with LPS (Eckel and Ametaj, 2016). The weaker immune response of dairy cows to LTA is also evident from tolerance of the udder and the reproductive system to chronic colonization by gram-positive bacteria, compared with a rapid and severe response to colonization/ infection by gram-negative bacteria in those organs (Strandberg et al., 2005; Gilbert et al., 2013).

Supplementation with SCFPb- $1 \times$ significantly reduced plasma concentrations of LTA compared with control cows ( 0.63 vs. $0.73 \mathrm{ng} / \mathrm{mL}$ ). We found no difference in plasma LTA concentration among SCFPb-1×, SCFPb- $2 \times$, and SCFPa. The reduced LTA concentration in SCFP-fed cows perhaps is an indicator of either less translocation of LTA endotoxins from the gut lumen and, hence, improved gut epithelial integrity compared with control cows, or a priming of the immune system in those animals (Mahmoud et al., 2020; Vailati-Riboni et al., 2021) for more effective clearance of LTA from circulation. Similar to LPS, LTA has been shown to disrupt barrier function and intestinal epithelial permeability via production of reactive oxygen species, in particular, inducible nitric oxide synthase, which increases the concentration of nitric oxide (Eckel and Ametaj, 2016). We attempted to measure LTA concentration in rumen fluid; however, the results were not reproducible (data not shown). We speculate that perhaps the presence of inhibitors in combination with greater concentrations of LTA in rumen fluid made it difficult to achieve proper dilutions, hence overwhelming the assay. To our knowledge, this is the first study to report LTA under SARA episodes.

\section{Effects of SARA and SCFP on Proinflammatory Cytokines (IL1- $\beta$ and IL-6) and APP (SAA and Hp) Concentrations in Peripheral Blood Plasma}

Both LPS and LTA bind to specific cell surface receptors to activate an endotoxin-associated immune response. Although LPS binds to CD14 and toll-like receptor (TLR) 4, LTA binds to CD14, CD36, and TLR2 to activate the release of proinflammatory mediators such as IL-1 $\beta$ and IL-6 (Dziarski et al., 2000; Ginsburg, 2002) and, subsequently, the production of acute-phase proteins, such as SAA, Hp, and LBP (Eckel and Ametaj, 2016). In this study, SARA challenges increased blood plasma concentrations of SAA and tended to increase that of IL- $1 \beta$ compared with Pre-SARA1, without affecting the plasma concentration of Hp and serum concentration of IL-6 (Table 4; Supplemental Tables S3, S6, and S7, https://figshare .com/articles/online_resource/Supplemental_data_for _DOI_10_3168_jds_2021-20572/17162603, Guo et al., 2021). Consistent with our results, the increase in plasma concentration of SAA following induction of SARA has been reported previously (Khafipour et al., 2009; Li et al., 2016; Zhao et al., 2018). Increases in serum concentrations of $\mathrm{Hp}$ and IL-1 $\beta$ were anticipated (Li et al., 2016; Zhao et al., 2018), whereas only a numerical increase or a tendency was observed, which could be because of moderate episodes of rumen $\mathrm{pH}$ depression. Although APP usually reach their peak concentration within days post-challenge, cytokine responses occur faster, reaching their peak within several hours postchallenge (Jacobsen et al., 2007; Paulina and Tadeusz, 2011). In this study, circulating variables were measured on d 2 and 5 following SARA induction; hence, it is possible that the window for proper monitoring of these cytokines was missed (Jacobsen et al., 2007). For instance, previously, with a more severe SARA induction model (313.5 vs. $186 \mathrm{~min} / \mathrm{d}$ of rumen $\mathrm{pH}<5.6)$ but a similar measurement time point, authors captured a tendency for increased IL-6 during SARA ( $\mathrm{Li}$ et al., 2016). Therefore, the lack of IL-6 response in the present study may be due to moderate episodes of rumen $\mathrm{pH}$ depression. These observations are also backed up by the lack of MPO elevation in rumen papillae, which is an indicator of tissue inflammation (Chami et al., 2018), supporting that, although we met the minimum requirements for induction of SARA, we did not induce a severe inflammation in rumen papillae. We should keep in mind, though, that MPO measurement was performed in papillae samples collected 2 wk after the second SARA challenge. As such, we may have similarly missed the window for proper monitoring of MPO.

From an immunological perspective, we consider an inflammatory response successful if the concentration of proinflammatory cytokines increases rapidly (speed of response) following the challenge and declines soon after achieving a peak (magnitude of response), to avoid unnecessary damage to the host (Jacobsen et al., 2007; Abbas et al., 2016). However, the magnitude of cytokine production can be reduced if or when immune cells are already primed (or trained) and have greater readiness and capacity to handle the immunogenic compounds or pathogenic organisms that threaten host health (Jacobsen et al., 2007; Vailati-Riboni et 
al., 2021). Here, across Pre-SARA, Post-SARA, and SARA stages, $\mathrm{SCFPb}-2 \times$ reduced plasma concentration of SAA and IL-1 $\beta$ and serum concentration of Hp compared with control-fed cows. These effects might be a result of greater priming effect of immune cells by SCFP, as reported previously. For instance, Mahmoud et al. (2020) reported that peripheral blood cells from SCFP-treated calves had an increased capacity for proinflammatory cytokine production in response to LPS stimulation and suggested that this innate training (or priming) has emerged as a mechanism by which cells of the innate immune system exist in an enhanced, or "trained," state, which enables them to respond to pathogenic and immunogenic insults with increased proinflammatory cytokine secretion.

\section{CONCLUSIONS}

Induction of 2 episodes of grain-based SARA challenges during early lactation increased free LPS concentrations in ruminal fluid and feces and tended to increase plasma concentration of LTA. Induction of SARA episodes increased concentration of circulating SAA and tended to increase that of IL- $1 \beta$ compared with Pre-SARA1, but did not affect the concentrations of circulating IL-6 and Hp. These data, together with undetectable plasma LPS and lack of rumen papillae MPO changes across all Pre-SARA, Post-SARA, and SARA stages, suggest that a moderate depression of rumen $\mathrm{pH}$ that slightly exceeded the SARA threshold promoted an inflammatory response but did not result in epithelial tissue damage. Supplementation of $\mathrm{SCFPb}$ at both $1 \times(19 \mathrm{~g} /$ cow per day $)$ and $2 \times$ doses attenuated the plasma concentration of LTA. Meanwhile, $\mathrm{SCFPb}$ supplementation at the $2 \times$ dose $(38 \mathrm{~g} / \mathrm{cow}$ per day) attenuated the free LPS concentrations of ruminal fluid compared with control in parity- 2 cows, and also reduced plasma concentration of SAA and serum concentration of IL-1 $\beta$ compared with control-fed cows. This could perhaps be suggestive of either less translocation of LTA and LPS endotoxins from the gut lumen and, hence, improved gut epithelial integrity associated with SCFPb feeding, or a greater priming of immune cells for more effective clearance of LTA and LPS from the systemic circulation.

\section{ACKNOWLEDGMENTS}

This study was supported by grants from Natural Science and Engineering Research Council (NSERC) of Canada Collaborative Research and Development (CRD) and Discovery programs, Dairy Farmers of Manitoba, Canada, and Diamond V (Cedar Rapids,
IA), to EK and JCP. The authors thank the staff of the Dairy Research Unit at the Glenlea Research Station, University of Manitoba (Glenlea, MB, Canada), for the maintenance and care of the animals. The authors also thank the members of Microbiome Laboratory at the University of Manitoba, including Behzad Kalantarpour, Paula Azevedo, Hooman Derakhshani, Hein Min Tun, and Shucong Li, for their assistance with laboratory and experimental set up. Ilkyu Yoon is the director of ruminant research at Diamond V. Ehsan Khafipour was an associate professor at the University of Manitoba when the study was conducted. He is currently the director of microbiome research at Cargill Health Technologies. The authors have not stated any other conflicts of interest.

\section{REFERENCES}

Abbas, A., A. H. Lichtman, and S. Pillai. 2016. Innate immunity. Pages 23-49 in Basic Immunology: Functions and Disorders of the Immune System. 9th ed. Elsevier.

Arroyo, J. M., A. Hosseini, Z. Zhou, A. Alharthi, E. Trevisi, J. S. Osorio, and J. J. Loor. 2017. Reticulo-rumen mass, epithelium gene expression, and systemic biomarkers of metabolism and inflammation in Holstein dairy cows fed a high-energy diet. J. Dairy Sci. 100:9352-9360. https://doi.org/10.3168/jds.2017-12866.

CCAC. 1993. Guide to the care and use of experimental animals. Vol. 1. Canadian Council on Animal Care. https://ccac.ca/en/ standards/guidelines/general-guidelines.html.

Chami, B., N. J. J. Martin, J. M. Dennis, and P. K. Witting. 2018. Myeloperoxidase in the inflamed colon: A novel target for treating inflammatory bowel disease. Arch. Biochem. Biophys. 645:61-71. https://doi.org/10.1016/j.abb.2018.03.012.

Chin, A. C., A. N. Flynn, J. P. Fedwick, and A. G. Buret. 2006. The role of caspase-3 in lipopolysaccharide-mediated disruption of intestinal epithelial tight junctions. Can. J. Physiol. Pharmacol. 84:1043-1050. https://doi.org/10.1139/y06-056.

Dziarski, R., A. J. Ulmer, and D. Gupta. 2000. Interactions of CD14 with components of gram-positive bacteria. Chem. Immunol. 74:83-107. https://doi.org/10.1159/000058761.

Eckel, E. F., and B. N. Ametaj. 2016. Invited review: Role of bacterial endotoxins in the etiopathogenesis of periparturient diseases of transition dairy cows. J. Dairy Sci. 99:5967-5990. https://doi.org/ 10.3168/jds.2015-10727.

Gilbert, F. B., P. Cunha, K. Jensen, E. J. Glass, G. Foucras, C. Robert-Granie, R. Rupp, and P. Rainard. 2013. Differential response of bovine mammary epithelial cells to Staphylococcus aureus or Escherichia coli agonists of the innate immune system. Vet. Res. 44:40. https://doi.org/10.1186/1297-9716-44-40.

Ginsburg, I. 2002. Role of lipoteichoic acid in infection and inflammation. Lancet Infect. Dis. 2:171-179. https://doi.org/10.1016/S1473 -3099(02)00226-8.

Gozho, G. N., D. O. Krause, and J. C. Plaizier. 2007. Ruminal lipopolysaccharide concentration and inflammatory response during grain-induced subacute ruminal acidosis in dairy cows. J. Dairy Sci. 90:856-866. https://doi.org/10.3168/jds.S0022-0302(07)71569 -2 .

Guo, J., Z. Zhang, H. Derakhshani, I. Yoon, J. C. Plaizier, and E. Khafipour. 2018. Effects of subacute ruminal acidosis (SARA) and Saccharomyces cerevisiae fermentation products on gastrointestinal microbiome of dairy cows. J. Anim. Sci. 96(Suppl. 3):398. https://doi.org/10.1093/jas/sky404.873.

Guo, J. L. Xu, H. Khalouei, K. B. Fehr, V. Senaratne, J.-E. Ghia, et al. 2021. Supplemental data for DOI https://doi.org/10.3168/jds 
.2021-20572. figshare. Online resource. https://doi.org/10.6084/ m9.figshare.17162603.v2.

Haubro Andersen, P., and N. Jarlov. 1990. Investigation of the possible role of endotoxin, TXA2, PGI2 and PGE2 in experimentally induced rumen acidosis in cattle. Acta Vet. Scand. 31:27-38. https: //doi.org/10.1186/BF03547574.

Humer, E., I. Kroger, V. Neubauer, K. Schedle, N. Reisinger, and Q. Zebeli. 2018. Supplementing phytogenic compounds or autolyzed yeast modulates ruminal biogenic amines and plasma metabolome in dry cows experiencing subacute ruminal acidosis. J. Dairy Sci. 101:9559-9574. https://doi.org/10.3168/jds.2018-14744.

Hurley, J. C. 1995. Endotoxemia: Methods of detection and clinical correlates. Clin. Microbiol. Rev. 8:268-292. https://doi.org/10 $.1128 /$ CMR. 8.2.268.

Jacobsen, S., P. H. Andersen, and B. Aasted. 2007. The cytokine response of circulating peripheral blood mononuclear cells is changed after intravenous injection of lipopolysaccharide in cattle. Vet. J. 174:170-175. https://doi.org/10.1016/j.tvjl.2006.06.006.

Jensen, G. S., K. M. Patterson, and I. Yoon. 2008. Yeast culture has anti-inflammatory effects and specifically activates nk cells. Comp. Immunol. Microbiol. Infect. Dis. 31:487-500.

Jiang, Y., I. M. Ogunade, D. H. Kim, X. Li, A. A. Pech-Cervantes, K. G. Arriola, A. S. Oliveira, J. P. Driver, L. F. Ferraretto, C. R. Staples, D. Vyas, and A. T. Adesogan. 2018. Effect of adding clay with or without a Saccharomyces cerevisiae fermentation product on the health and performance of lactating dairy cows challenged with dietary aflatoxin b1. J. Dairy Sci. 101:3008-3020. https://doi .org/10.3168/jds.2017-13678.

Khafipour, E., D. O. Krause, and J. C. Plaizier. 2009. A grain-based subacute ruminal acidosis challenge causes translocation of lipopolysaccharide and triggers inflammation. J. Dairy Sci. 92:10601070. https://doi.org/10.3168/jds.2008-1389.

Khafipour, E., S. Li, H. M. Tun, H. Derakhshani, S. Moossavi, and J. C. Plaizier. 2016. Effects of grain feeding on microbiota in the digestive tract of cattle. Anim. Front. 6:13-19. https://doi.org/10 .2527 /af.2016-0018.

Khalouei, H., V. Seranatne, K. B. Fehr, J. Guo, I. Yoon, E. Khafipour, and J. C. Plaizier. 2021. Effects of Saccharomyces cerevisiae fermentation products and subacute ruminal acidosis on feed intake, fermentation, and nutrient digestibilities in lactating dairy cows. Can. J. Anim. Sci. 101:143-157. https://doi.org/10.1139/cjas-2020 $-0018$.

Khiaosa-Ard, R., and Q. Zebeli. 2018. Diet-induced inflammation: From gut to metabolic organs and the consequences for the health and longevity of ruminants. Res. Vet. Sci. 120:17-27. https://doi .org/10.1016/j.rvsc.2018.08.005.

Krause, K. M., and G. R. Oetzel. 2006. Understanding and preventing subacute ruminal acidosis in dairy herds: A review. Anim. Feed Sci. Technol. 126:215-236. https://doi.org/10.1016/j.anifeedsci .2005.08.004.

Li, S., E. Khafipour, D. O. Krause, A. Kroeker, J. C. RodriguezLecompte, G. N. Gozho, and J. C. Plaizier. 2012. Effects of subacute ruminal acidosis challenges on fermentation and endotoxins in the rumen and hindgut of dairy cows. J. Dairy Sci. 95:294-303. https://doi.org/10.3168/jds.2011-4447.

Li, S., E. Khafipour, D. O. Krause, J. C. Rodriguez-Lecompte, and J. C. Plaizier. 2010. Free endotoxins in the feces of lactating dairy cows. Can. J. Anim. Sci. 90:591-594. https://doi.org/10.4141/ cjas10017.

Li, S., I. Yoon, M. Scott, E. Khafipour, and J. C. Plaizier. 2016. Impact of Saccharomyces cerevisiae fermentation product and subacute ruminal acidosis on production, inflammation, and fermentation in the rumen and hindgut of dairy cows. Anim. Feed Sci. Technol. 211:50-60. https://doi.org/10.1016/j.anifeedsci.2015.10.010.

Mahmoud, A. H. A., J. R. Slate, S. Hong, I. Yoon, and J. L. Mcgill. 2020. Supplementing a Saccharomyces cerevisiae fermentation product modulates innate immune function and ameliorates bovine respiratory syncytial virus infection in neonatal calves. J. Anim. Sci. 98:skaa252. https://doi.org/10.1093/jas/skaa252.
Mao, S. Y., R. Y. Zhang, D. S. Wang, and W. Y. Zhu. 2013. Impact of subacute ruminal acidosis (SARA) adaptation on rumen microbiota in dairy cattle using pyrosequencing. Anaerobe 24:12-19. https://doi.org/10.1016/j.anaerobe.2013.08.003.

Monteiro, H. F., and A. P. Faciola. 2020. Ruminal acidosis, bacterial changes, and lipopolysaccharides. J. Anim. Sci. 98:skaa248. https: //doi.org/10.1093/jas/skaa248.

Paulina, J., and S. Tadeusz. 2011. Acute phase proteins in cattle. In Acute Phase Proteins as Early Non-Specific Biomarkers of Human and Veterinary Diseases. F. Veas, ed. IntechOpen.

Perdomo Lozada, M. C. 2011. Dietary strategies to modulate performance, health, and immune responses in Holstein calves. $\mathrm{PhD}$, Department of Animal Science, Institute for Food and Agricultural Sciences, University of Florida. Gainesville.

Petri, R. M., T. Schwaiger, G. B. Penner, K. A. Beauchemin, R. J. Forster, J. J. Mckinnon, and T. A. Mcallister. 2013. Characterization of the core rumen microbiome in cattle during transition from forage to concentrate as well as during and after an acidotic challenge. PLoS One 8:e83424. https://doi.org/10.1371/journal.pone .0083424 .

Plaizier, J., E. Khafipour, S. Li, G. Gozho, and D. Krause. 2012. Subacute ruminal acidosis (SARA), endotoxins and health consequences. Anim. Feed Sci. Technol. 172:9-21. https://doi.org/10 .1016/j.anifeedsci.2011.12.004.

Plaizier, J. C., A. M. Danscher, P. A. Azevedo, H. Derakhshani, P. H. Andersen, and E. Khafipour. 2021. A grain-based SARA challenge affects the composition of epimural and mucosa-associated bacterial communities throughout the digestive tract of dairy cows. Animals (Basel) 11:1658. https://doi.org/10.3390/ani11061658.

Poppy, G. D., A. R. Rabiee, I. J. Lean, W. K. Sanchez, K. L. Dorton, and P. S. Morley. 2012. A meta-analysis of the effects of feeding yeast culture produced by anaerobic fermentation of Saccharomyces cerevisiae on milk production of lactating dairy cows. J. Dairy Sci. 95:6027-6041. https://doi.org/10.3168/jds.2012-5577.

Qumar, M., R. Khiaosa-ard, F. Klevenhusen, J. C. Plaizier, and Q. Zebeli. 2017. Gastrointestinal endotoxin and metabolic responses in cows fed and recovered from two different grain-rich challenges. Livest. Sci. 203:120-123. https://doi.org/10.1016/j.livsci.2017.07 .015 .

Salminen, S., M. C. Collado, A. Endo, C. Hill, S. Lebeer, E. M. M. Quigley, M. E. Sanders, R. Shamir, J. R. Swann, H. Szajewska, and G. Vinderola. 2021. The International Scientific Association of Probiotics and Prebiotics (ISAPP) consensus statement on the definition and scope of postbiotics. Nat. Rev. Gast. Hepat. 18:649667. https://doi.org/10.1038/s41575-021-00440-6.

Steele, M. A., O. Alzahal, S. E. Hook, J. Croom, and B. W. Mcbride. 2009. Ruminal acidosis and the rapid onset of ruminal parakeratosis in a mature dairy cow: A case report. Acta Vet. Scand. 51:39. https://doi.org/10.1186/1751-0147-51-39.

Strandberg, Y., C. Gray, T. Vuocolo, L. Donaldson, M. Broadway, and R. Tellam. 2005. Lipopolysaccharide and lipoteichoic acid induce different innate immune responses in bovine mammary epithelial cells. Cytokine 31:72-86. https://doi.org/10.1016/j.cyto.2005.02 .010 .

Trevisi, E., N. Jahan, G. Bertoni, A. Ferrari, and A. Minuti. 2015. Pro-inflammatory cytokine profile in dairy cows: Consequences for new lactation. Ital. J. Anim. Sci. 14:3862. https://doi.org/10 .4081/ijas.2015.3862.

Tun, H. M., S. Li, I. Yoon, S. J. Meale, P. Azevedo, E. Khafipour, and J. C. Plaizier. 2020. Saccharomyces cerevisiae fermentation products (SCFP) stabilize the ruminal microbiota of lactating dairy cows during periods of a depressed rumen $\mathrm{pH}$. BMC Vet. Res. 16:237. https://doi.org/10.1186/s12917-020-02437-w.

Vailati-Riboni, M., D. N. Coleman, V. Lopreiato, A. Alharthi, R. E. Bucktrout, E. Abdel-Hamied, I. M. Cortes, E. Trevisi, I. Yoon, and J. J. Loor. 2021. Feeding a Saccharomyces cerevisiae fermentation product improves udder health and immune response to a Streptococcus uberis mastitis challenge in mid-lactating dairy cows. J. Anim. Sci. Biotechnol. 12:62. https://doi.org/10.1186/s40104 -021-00560-8. 
Weber, J. R., P. Moreillon, and E. I. Tuomanen. 2003. Innate sensors for gram-positive bacteria. Curr. Opin. Immunol. 15:408-415. https://doi.org/10.1016/S0952-7915(03)00078-5.

Zhang, R. Y., I. Yoon, W. Y. Zhu, and S. Y. Mao. 2013. Effect of Saccharomyces cerevisiae fermentation product on lactation performance and lipopolysaccharide concentration of dairy cows. AsianAustralas. J. Anim. Sci. 26:1137-1143. https://doi.org/10.5713/ ajas.2013.13181.

Zhao, C., G. Liu, X. Li, Y. Guan, Y. Wang, X. Yuan, G. Sun, Z. Wang, and X. Li. 2018. Inflammatory mechanism of rumenitis in dairy cows with subacute ruminal acidosis. BMC Vet. Res. 14:135. https: //doi.org/10.1186/s12917-018-1463-7.

Zhu, W., Z. Wei, N. Xu, F. Yang, I. Yoon, Y. Chung, J. Liu, and J. Wang. 2017. Effects of Saccharomyces cerevisiae fermentation products on performance and rumen fermentation and microbiota in dairy cows fed a diet containing low quality forage. J. Anim. Sci. Biotechnol. 8:36. https://doi.org/10.1186/s40104-017-0167-3.

\section{ORCIDS}

J. Guo $\odot$ https://orcid.org/0000-0001-7903-8252

V. Senaratne ำ https://orcid.org/0000-0001-5428-6946

I. Yoon () https://orcid.org/0000-0003-1891-1585

E. Khafipour ๑ https://orcid.org/0000-0003-4673-7633

J. C. Plaizier (1) https://orcid.org/0000-0002-8551-9535 